\title{
Determination of Metallic Impurities in a Silicon Wafer by Local Etching and Electrothermal Atomic Absorption Spectrometry
}

\author{
Hye-Young Chung, *广 Sang-Hak LeE, ** Young-Hun KIM,* Ki-Sang LEE,* and Dae-Hong KIM* \\ $* R$ \& D Center, LG Siltron Inc., 283 Imsoo-dong, Gumi, 730-350, Korea \\ **Department of Chemistry, Kyungpook National University, 1370 Sankyuk-dong, Puk-gu, \\ Daegu 702-701, Korea
}

\begin{abstract}
An etching technique for the determination of the metallic impurities distribution in silicon wafers has been developed. An area of $10 \mathrm{~mm} \phi$ and $10 \mu \mathrm{m}$ depth was etched by $100 \mu \mathrm{L}$ of an etching solution with a $\mathrm{HF}$ and $\mathrm{HNO}_{3}$ mixture. The acid matrix was evaporated on the wafer surface by IR lamp illumination and vacuum exhaust. Metallic impurities remaining on the wafer surface were redissolved into the collection solution, which was measured by electrothermal atomic absorption spectrometry (ET-AAS). The recovery invested by local etching/ET-AAS was within $95-112 \%$ for $\mathrm{Fe}, \mathrm{Cu}$ and $\mathrm{Ni}$. The detection limit $(3 \sigma)$ for $\mathrm{Fe}, \mathrm{Cu}$ and $\mathrm{Ni}$ in silicon was $1 \times 10^{13}$ atoms $/ \mathrm{cm}^{3}$. To confirm the applicability, local etching was applied to evaluate the effects of metallic impurities in a gettering study and the electronic properties of semiconductor devices. It was found that local etching is a useful sample preparation technique for the analysis of metallic impurities in a specific area on a silicon wafer.
\end{abstract}

(Received November 28, 2002; Accepted May 8, 2003)

\section{Introduction}

As the packing density increases in an ultra-large-scale integrated (ULSI) device manufacturing process, the requirement of an acceptable metallic contamination level becomes more stringent. ${ }^{1}$ Because trace amounts of metallic impurities affect the performance of electronic devices, a highly precise analytical technique is demanded. Vapor-phase decomposition (VPD) sample preparation is one of the most prevalent analytical methods for metallic impurity analysis., ${ }^{2,3}$ Oxides on a silicon wafer are decomposed with HF vapor, or mixed acid vapor, and then a liquid droplet is rolled on the hydrophobic surface. VPD preparation has been coupled with several different trace elemental analytical techniques, such as inductively coupled plasma mass spectrometry (ICP-MS), ${ }^{4-7}$ atomic absorption spectrometry (AAS) $)^{8-11}$ and total-reflection $\mathrm{X}$-ray fluorescence (TXRF). ${ }^{12-14}$

In order to analyze the metallic impurities in a bulk of silicon wafer, etching or bulk decomposition sample preparation is used. $^{15,16}$ The whole wafer surface is etched toward depth, or a silicon specimen is decomposed by acids. Then the decomposed silicon and acids are removed by heating on a hot plate. Metallic impurities remaining after matrix removal are measured by analytical instruments. The results from these methods, such as VPD preparation, etching or bulk decomposition, are shown as the mean value of the entire wafer surface or bulk. Metallic impurities, however, are not contaminated uniformly in a wafer in the device or wafer manufacturing process. In addition, ultra-trace metallic impurities are not detected by the dilution effect of a large sampling size. Therefore, there is a great demand to analyze

$\dagger$ To whom correspondence should be addressed.

E-mail: jhyc@mail.lgsiltron.co.kr trace metallic impurities for a specific area.

In this paper, the analytical method for a specific area on a wafer is described. For the sample preparation of a local area and a specific depth of a silicon wafer, a sampling apparatus has been developed. The etching area can be easily defined using this apparatus at the same fixed positions on several wafers. After preparation, $\mathrm{Fe}, \mathrm{Cu}$ and $\mathrm{Ni}$ were measured by the ETAAS. Determination by ICP-MS or ET-AAS is a common technique for the analysis of metallic impurities. Even though ICP-MS has a lower detection limit, it needs a special sample introduction system for a small-volume sample containing $\mathrm{Si}$ and HF. Because of a few instrumental limitations, ET-AAS was used in this experiment. As an application using a local etching apparatus, wafers annealed after the contamination of metallic impurities and patterned wafers were analyzed.

\section{Experimental}

\section{Instrument and apparatus}

A Perkin Elmer ZL5100 GF-AAS (Perkin Elmer, Norwalk, CT, USA) equipped with a Zeeman background corrector was used to measure $\mathrm{Fe}, \mathrm{Cu}$ and $\mathrm{Ni}$. A pyrocoated graphite tube was used, the operating conditions are described in Table 1. For sample preparation, the etching apparatus is made with polytetrafluoroethylene (PTFE). A schematic diagram of the etching apparatus is shown in Fig. 1.

The apparatus is divided into three parts: main part, IR lamp part and vacuum exhausting part. The main part is composed of a main plate and a cover plate with punched holes. Also, an IR lamp is set for heating the etching solution, and the reaction gas is vented by the vacuum exhausting part. The diameter of the sampling tube is $10 \mathrm{~mm} \phi$. The sampling tube is designed especially for protecting the leakage of the etching solution while the silicon wafer is being etched. 
Table 1 Operating conditions of electrothermal AAS

\begin{tabular}{|c|c|c|c|c|c|c|c|c|c|}
\hline \multirow{2}{*}{$\begin{array}{l}\text { Graphite furnace program } \\
\text { Step }\end{array}$} & \multicolumn{3}{|c|}{$\mathrm{Fe}$} & \multicolumn{3}{|c|}{$\mathrm{Cu}$} & \multicolumn{3}{|c|}{$\mathrm{Ni}$} \\
\hline & $\begin{array}{c}\text { Temp. } \\
/{ }^{\circ} \mathrm{C}\end{array}$ & $\begin{array}{l}\text { Ramp } \\
\text { time/s }\end{array}$ & $\begin{array}{l}\text { Hold } \\
\text { time/s }\end{array}$ & $\begin{array}{c}\text { Temp. } \\
/^{\circ} \mathrm{C}\end{array}$ & $\begin{array}{l}\text { Ramp } \\
\text { time/s }\end{array}$ & $\begin{array}{c}\text { Hold } \\
\text { time/s }\end{array}$ & $\begin{array}{l}\text { Temp. } \\
/{ }^{\circ} \mathrm{C}\end{array}$ & $\begin{array}{l}\text { Ramp } \\
\text { time/s }\end{array}$ & $\begin{array}{c}\text { Hold } \\
\text { time/s }\end{array}$ \\
\hline Drying & 110 & 1 & 30 & 110 & 5 & 30 & 110 & 20 & 30 \\
\hline \multirow[t]{2}{*}{ Ashing } & 130 & 15 & 30 & 130 & 15 & 35 & 130 & 25 & 35 \\
\hline & 800 & 10 & 20 & 1200 & 15 & 20 & 1300 & 10 & 20 \\
\hline Atomization & 2300 & 0 & 5 & 2300 & 0 & 5 & 2400 & 0 & 5 \\
\hline Cleaning & 2500 & 1 & 2 & 2550 & 1 & 2 & 2500 & 1 & 2 \\
\hline Sample/ $\mu \mathrm{L}$ & & 30 & & & 30 & & & 40 & \\
\hline Wavelength/nm & & 248.3 & & & 324.8 & & & 232.0 & \\
\hline
\end{tabular}

Background correction, Zeeman effect background correction; signal measurement, peak height mode.

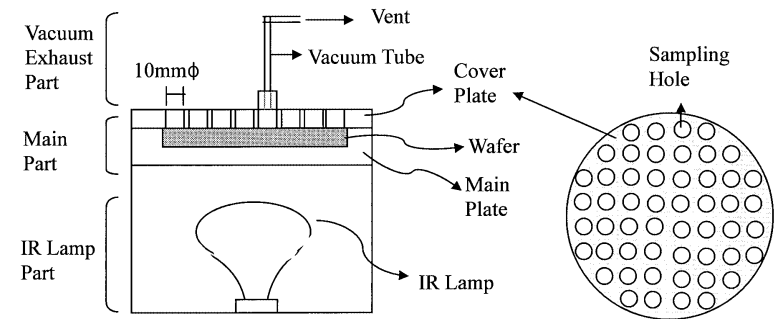

Fig. 1 Schematic diagram of the local etching apparatus.

\section{Reagents and samples}

$\mathrm{HNO}_{3}(55 \mathrm{wt} \%)$, $\mathrm{HF}(38 \mathrm{wt} \%)$ and $\mathrm{H}_{2} \mathrm{O}_{2}(35 \mathrm{wt} \%)$ of a highpurity solution (TAMAPURE AA-10, Tama Chemical Co., Kanagawa, Japan) were used in all of the experiments. Stock standard solutions $\left(1000 \mu \mathrm{g} \mathrm{mL}^{-1}\right)$ of High Purity Standards (Charleston, SC, USA) were used. Working standard solutions of $\mathrm{Fe}, \mathrm{Cu}$ and $\mathrm{Ni}$ were prepared prior to use by diluting $1.0 \mu \mathrm{g}$ $\mathrm{mL}^{-1}$ of the corresponding standard solution with $2 \% \mathrm{HNO}_{3}$. Samples were boron-doped wafers with a diameter of $200 \mathrm{~mm}$ and single-side polished and cleaned with VPD sample preparation prior to use.

\section{Procedure}

A wafer was placed between the main plate and the cover plate. A sampling tube was placed in a hole at a specific location for the analysis. One hundred microliters of the etching solution were dropped onto the wafer surface encircled by the sampling tube. A vacuum tube was connected in the hole of the sampling tube. The reaction gas was exhausted by a vacuum pump, and the etching solution was dried by illuminating an IR lamp under the wafer. After drying etching solution, the cover plate and the vacuum tube were removed. Two hundred microliters of a dissolution solution with $1 \% \mathrm{H}_{2} \mathrm{O}_{2}$ and $0.2 \% \mathrm{HF}$ were pipetted into the sampling tube. This composition of the dissolution solution was shown to be the optimum condition to collect metallic impurities on the wafer surface in a previous report. ${ }^{3}$ The dissolution solution was collected by a micropipette after one min. The collected solution was measured by ET-AAS. All of the experiments were carried out in a class-100 clean room.
Table 2 Etched depth of a silicon wafer based on the composition of the etching solutions

\begin{tabular}{crcc}
\hline $\begin{array}{c}\text { Etching solution } \\
\mathrm{HF} \%: \mathrm{HNO}_{3} \%\end{array}$ & $\begin{array}{c}\text { Etched depth } / \\
\mu \mathrm{m}\end{array}$ & $\begin{array}{c}\text { Etched area/ } \\
\mathrm{cm}^{2}\end{array}$ & $\begin{array}{c}\text { Volume of etching } \\
\text { solution/ } \mu \mathrm{L}\end{array}$ \\
\hline $2: 25$ & $5.2 \pm 0.2$ & 0.785 & 100 \\
$5: 25$ & $10.3 \pm 0.6$ & & \\
$10: 25$ & $19.0 \pm 0.5$ & & \\
\hline
\end{tabular}

a. Mean \pm standard deviation, $n=3$.

\section{Results and Discussion}

\section{Optimization of the local etching conditions}

The etching solution was fixed as a mixture of $\mathrm{HF}$ and $\mathrm{HNO}_{3}$ that has been used as an etching solution of silicon in the semiconductor industry for a long time. The volume of the etching solution was limited to $100 \mu \mathrm{L}$ so as to avoid any leakage of chemicals during the etching reaction and the long dry time needed to remove the solution when using a large volume. The desired etching depth was $10 \mu \mathrm{m}$. A device pattern is usually fabricated within $10 \mu \mathrm{m}$ depth from the surface of the silicon wafer. Therefore, we have been concerned about metallic impurities at this depth. Table 2 shows the etched depth of a silicon wafer produced by different compositions of the etching solution (HF: $2 \%, 5 \%$ and 10\%). An etched depth of $10 \mu \mathrm{m}$ was obtained when the concentration of $\mathrm{HF}$ was $5 \%$. The thickness of the wafer before and after etching was measured by a thickness gauge instrument from Tokyo Seimitsu (A070145, Tokyo Seimitsu, Japan). The etching reaction was completed after $20 \mathrm{~min}$.

\section{Recovery test for a local etching procedure}

A recovery test was carried out at the optimal etching conditions. One hundred microliters of a $1.0 \mathrm{ng} \mathrm{mL}^{-1}$ standard solution of $\mathrm{Fe}, \mathrm{Cu}$ and $\mathrm{Ni}$ were pipetted onto the wafer surface, and dried by an IR lamp. The area containing a contaminated spot was analyzed with a local etching technique. The recovery was determined as the ratio of the measured concentration to the initial contaminated concentration. A recovery of $95-112 \%$ was obtained from the mean of five samples, as tabulated in Table 3.

The detection limits obtained in this experiment are also given in Table 3. They were calculated using three-times the standard deviation of the blank solution, which was treated with the same procedure without spiking. 
Table 3 Recovery results of the spiked and detection limits obtained by local etching/AAS

\begin{tabular}{ccc}
\hline & Recovery, $\%$ & Detection limit, atom $/ \mathrm{cm}^{3}$ \\
\hline $\mathrm{Cu}$ & $102 \pm 22$ & $6.6 \mathrm{E}+13$ \\
$\mathrm{Fe}$ & $95 \pm 9$ & $9.2 \mathrm{E}+13$ \\
$\mathrm{Ni}$ & $112 \pm 13$ & $7.5 \mathrm{E}+13$ \\
\hline
\end{tabular}

$100 \mathrm{ng}$ of each element was spiked. Mean \pm standard deviation, $n=$ 9.

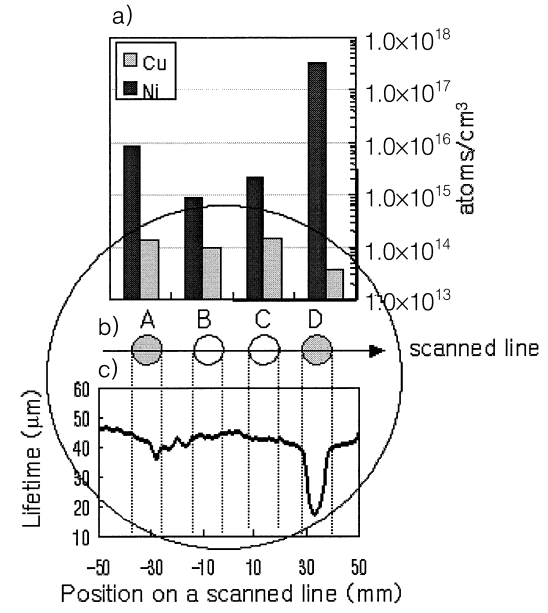

Fig. 2 Analysis results of $\mathrm{Cu}$ and $\mathrm{Ni}$ on a wafer: (a) local etching/AAS result, (b) contaminated and measured position of the wafer, (c) $\mu$-PCD lifetime result.

\section{Applications in real samples}

This local etching technique was applied to a $\mathrm{Ni}$ and $\mathrm{Cu}$ contaminated wafer for a gettering study of a silicon wafer. For this study, small amounts of $\mathrm{Cu}$ and $\mathrm{Ni}$ contaminant solutions were dropped onto arbitrary positions of the wafer surface and heat-treated for diffusion to the wafer bulk. The contaminated positions on a wafer and the measured result using a local etching technique are shown in Fig. 2. The A and D positions were contaminated with $\mathrm{Cu}$ and $\mathrm{Ni}$ with $500 \mu \mathrm{L}$ of $1 \mu \mathrm{g} \mathrm{mL} \mathrm{m}^{-1}$, respectively. The $\mathrm{B}$ and $\mathrm{C}$ areas were not contaminated for a reference. The $\mu$-photoconductance decay (PCD) lifetime measured by a lifetime scanner (WT-85X, Semilab, Hungary) decreased in the Ni contaminated area (D) compared with the non-contaminated area (B and C). At area D, where the $\mu$-PCD lifetime decreased, $\mathrm{Ni}$ was detected on the order of $2 \times 10^{17}$ atoms $/ \mathrm{cm}^{3}$ by the conversed unit in silicon. However, neither a decrease in the lifetime nor the detection of $\mathrm{Cu}$ using local etching was found in the $\mathrm{Cu}$ contaminated area (A). This means that $\mathrm{Cu}$ has an electrically inactive status, and does not exist in the device active area. From this result, we can predict the effect of metallic impurities after a heat treatment. Therefore, it is believed that a local etching technique played a useful role to interpret the behavior of metallic impurities in silicon and problems caused by metallic contamination.

As another application, a local etching technique was applied to a metal oxide semiconductor (MOS) patterned wafer. $\mathrm{Cu}$ was intentionally contaminated on the wafer surface by a dipping method. In this as-contaminated state, the total adsorbed $\mathrm{Cu}$ was measured to an order of $5 \times 10^{11}$ atoms $/ \mathrm{cm}^{2}$ on the wafer surface. MOS capacitors of $12 \mathrm{~nm}$ oxide were
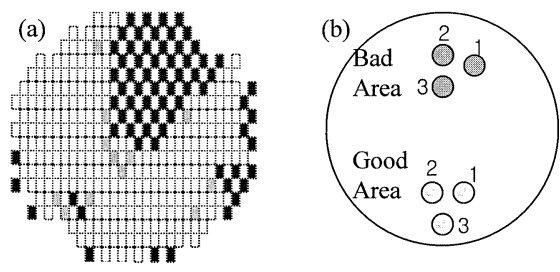

Fig. 3 BV failure distribution of a $\mathrm{Cu}$ contaminated wafer: (a) failure distribution map, (b) local etching/AAS sampling position.

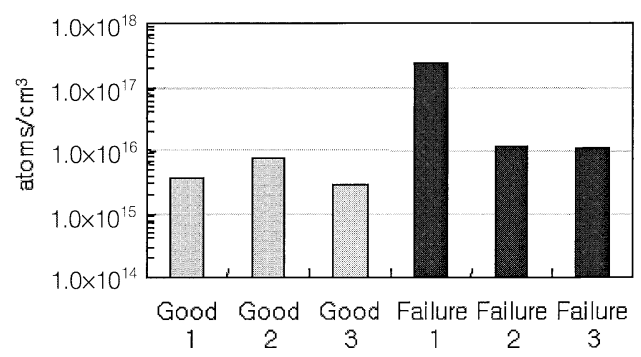

Fig. 4 Analysis result of $\mathrm{Cu}$ on the $\mathrm{BV}$ failure positions.

patterned, and the oxide breakdown voltage (BV) was measured. In Fig. 3, the electrically bad and good areas are distinguished as black and white rectangles. The criteria of the $\mathrm{BV}$ failure was $8 \mathrm{MV} / \mathrm{cm}$. Eventually, the BV results showed particular area trends in the fail distribution. A much greater BV failure occurred in the upper-side region of the wafer map, compared to other regions. It is presumed that surface condition would be varied along the dipping direction in which wafer was handled into/out of a solution. It was reported that the adsorption rates of $\mathrm{Cu}$ ion was influenced by the hydrophilicity and surface potential. ${ }^{17,18}$ Three points at good and bad areas were analyzed using a local etching technique. As shown in Fig. 4, $\mathrm{Cu}$ detection in the bad areas was relatively higher than that in the good areas. Stacking-fault-like defects were observed on the etched surface of the bad area. In other work, it was reported that these stacking-fault-like defects were generated by $\mathrm{Cu}$ contamination. ${ }^{19}$

It was demonstrated that metallic impurities on a specific area could be effectively analyzed using a local etching technique. The greatest advantage of the local etching apparatus would be its universal applicability to diverse wafer samples, such as polished, oxidized and patterned wafers. If the conditions of local etching, such as chemical composition or concentration, etching area and etching depth are controlled, the local etching technique could be used to analyze not only the effect of metallic impurities on the electronic property of semiconductor devices, but also trace metallic impurities in various device layers.

\section{References}

1. W. Kern, in "Handbook of Semiconductor Wafer Cleaning Technology", ed. W. Kern, 1992, Noyes Publications, Park Ridge, NJ, 596.

2. R. S. Hockett, in "Handbook of Semiconductor Wafer Cleaning Technology", ed. W. Kern, 1992, Noyes Publications, Park Ridge, NJ, 584. 
3. H. Y. Chung, Y. H. Kim, H. Y. Cho, B. Y. Lee, H. D. Yoo, and S. H. Lee, Anal. Sci., 2001, 17, 653.

4. M. Takenaka, Y. Yamada, M. Hayashi, H. Omori, S. Ito, and A. Okada, Bunseki Kagaku, 1997, 46, 743.

5. G. Settembre and E. Debrah, Micro, 1998, 16, 79.

6. K. Fujiwara, Y. Toumori, H. Mitsumata, M. Inada, and T. Nakahara, Bunseki Kagaku, 1999, 48, 681.

7. M. Komoda, K. Chiba, and H. Uchida, Anal. Sci., 1996, 12, 21.

8. H. Nagasawa and M. Enomoto, Bunseki Kagaku, 1997, 46, 375.

9. L. H. Hall, J. A. Sees, and B. L. Schmidt, Surface Interface Anal., 1996, 24, 511.

10. A. Shimazaki, H. Hiratsuka, Y. Matsushita, and S. Yoshii, in Extended Abstracts of the 16th Conference on Solid State Devices and Materials, Jpn. Soc. of Appl. Phys., Kobe, 1984, 281 - 284.

11. Y. Tanizoe, S. Sumita, M. Sano, N. Fujino, and T. Shiraiwa, Bunseki Kagaku, 1989, 34, 177.

12. M. Yamagami, M. Nonoguchi, T. Yamada, T. Shoji, T. Utaka, Y. Mori, S. Nomura, K. Taniguchi, H. Wakata, and
S. Ikeda, Bunseki Kagaku, 1999, 48, 1005.

13. R. S. Hockett, Anal. Sci., 1995, 11, 511.

14. S. Pahike, L. Kotz, T. Ehmann, P. Eichinger, and A. Huber, in Proceedings of the Eighth International Symposium on Silicon Materials Science and Technology, ed. H. R. Huff, H. Ysuya, and U. Gösele, ECS, San Diego, California, 1998, 1524 - 1540.

15. M. B. Shabani, T. Yoshimi, and H. Abe, J. Electrochem. Soc., 1996, 143, 2025.

16. Y. H. Kim, K. S. Lee, H. Y. Chung. D. H. Hwang, H. S. Kim, H. Y. Cho, and B. Y. Lee, J. Kor. Phy. Soc., 2001, 39, S348.

17. S.-W. Lim, R. T. Mo, P. A. Pianetta, and C. E. D. Chidsey, J. Electrochem. Soc., 2001, 148, C16.

18. K. K. Yineshige, H. G. Parks, S. Kaghavan, J. B. Hiskey, and P. J. Resnick, J. Electrochem. Soc., 1995, 142, 671.

19. M. Takiyama, S. Ohtsuka, S. Hayashi, and M. Tachimori, in Proceedings of the Seventh International Symposium on Silicon Materials Science and Technology, ed. H. R. Huff, W. Bergholz, and K. Sumino, ECS, San Francisco, 1994, $346-357$. 